\title{
PRECIO DE LA HARINA DE PESCADO Y EL VALOR ECONÓMICO AGREGADO EN AUSTRAL GROUP SAA 2009-2018
}

\author{
PRICE OF FISHMEAL AND THE ECONOMIC VALUE \\ ADDED IN AUSTRAL GROUP SAA 2009-2018
}

\author{
Recepción: 2020-03-26 - Aceptación: 2020-11-19
}

\author{
Juan Carlos Ocampo-Risco ${ }^{1 ;}$ a \\ (iD) ORCID iD 0000-0003-0851-7488 \\ ${ }^{1}$ Universidad Privada del Norte. \\ a Licenciado en Economía.
}

\section{RESUMEN}

Objetivo: Determinar si la relación que existe entre el precio de la harina de pescado y el valor económico agregado- EVA en Austral Group SAA cumple los postulados básicos de la teoría financiera. Métodos: Se utiliza series de tiempo económicas para determinar la relación econométrica entre el precio internacional de la harina de pescado y la generación de valor económico agregado en Austral Group SAA. Resultados: Respecto al precio de la harina y el valor económico agregado, se encuentra la relación negativa entre ambas variables con un alto nivel de significancia para el precio ( $p$-value $<10 \%$ ), otras variables significativas son la inversión de capital y el nivel de captura, pero con relación positiva. Conclusiones: El precio de la harina es componente del valor de las exportaciones, pero también de los costos de la empresa Austral Group SAA y demás empresas del sector. Un mayor precio de la harina eleva los derechos de pesca, se deben reformular estos pagos para reducir la relación negativa.

Palabras clave: Gestión financiera; EVA; Precio; Harina de pescado; Pesquería; Inversión.

\section{ABSTRACT}

Objective: Determine if the relationship between the price of fishmeal and the economic value added-EVA in Austral Group SAA meets the basic assumptions of financial theory. Methods: Economic time series are used to determine the econometric relationship between the international price of fishmeal and the generation of economic value added in Austral Group SAA. Results: Regarding the price of flour and the economic value added, there is a negative relationship between the former and the latter with a high level of significance for the price ( $p$-value $<10 \%$ ), other significant variables are capital investment and the level of capture but with a positive relationship. Conclusions: The price of fishmeal is a component of the value of exports, but also of the costs of Austral Group SAA and other companies in the sector. A higher price of fishmeal raises fishing rights, these fees and charges should be reformulated to reduce the negative relationship.

Keywords: Financial management; EVA, Price; Fishmeal; Fishery; Investment. 


\section{INTRODUCCIÓN}

Uno de los aspectos a los cuales más atención prestan los gestores financieros es la fijación del precio de sus productos; se espera que cuanto mayor sea este -ceteris paribus- mayor será la creación de valor para los accionistas, definida como los beneficios económicos obtenidos por encima de una inversión con riesgo equivalente. En este trabajo de investigación se trata de establecer la validez empírica de tal postulado; con este objetivo se trata de contestar a la interrogante de si el incremento en los precios internacionales de la harina de pescado ha implicado una mayor creación de valor en la pesquera Austral Group SAA (AGSAA).

Amat (2017) señala que el valor económico agregado es una herramienta que permite calcular y evaluar la riqueza generada por la empresa, este instrumento toma en cuenta el nivel de riesgo. Este autor indica que se crea valor cuando el beneficio operativo supera el costo de oportunidad de los activos invertidos. Se considera una destrucción de valor cuando el EVA es negativo.

Álvarez (2016) señala que EVA es el valor de la utilidad operacional luego de impuestos restando el capital invertido necesario para generar el beneficio. El valor económico añadido, enfoca a las empresas a la distribución adecuada de recursos, la generación de reportes específicos y significativos de desempeño.

En Perú, Chu (2015) señala que la información contable (histórica, bajo las normas internacionales de contabilidad) para medir el desempeño de la creación de valor tiene limitaciones, por cuanto solo son fundamentales en un período determinado.

La industria pesquera peruana posee recursos muy valiosos para la seguridad alimentaria del mundo. La FAO (Estado Mundial de la Pesca 2020) observa una disminución de la captura de peces en aguas continentales y un aumento de la acuicultura. Existe una gran necesidad por el consumo de alimentos con alto contenido de proteína, siendo la anchoveta un recurso estratégico para el desarrollo de la acuicultura, la industria porcina y avícola.

Para una mejor gestión financiera no son suficientes utilidades crecientes, se requiere crear valor y para tal fin, los beneficios deben ser mayores al costo de oportunidad de los recursos invertidos. Gómez-Bezares, Madariaga y Santibáñez (2013) señalan que el concepto del Valor Económico Agregado (EVA), es la reinvención de un viejo concepto, el Beneficio Económico; en este sentido, proponen que los beneficios no se deben reducir a lo contable, sino que debe considerarse el costo de oportunidad del capital invertido. Si el beneficio contable no alcanza para cubrir el costo de oportunidad del capital invertido, no habrá creación de valor. En particular, proponen que los beneficios antes de intereses, pero netos de impuestos, deben ser mayores al capital invertido multiplicado por el costo de oportunidad correspondiente. A partir de los estados financieros, los autores derivan la siguiente fórmula para la creación de valor:

$$
B E=(r-k) \cdot(A F+F M)
$$

Donde $r$ Rendimiento operativo sobre activos.

k Coste fijo de mediación.

AF Activo fijo

FM Fondo de Maniobra o capital de trabajo

BE Beneficio económico

Como se aprecia en la ecuación previa, existirá creación de valor si los rendimientos operativos son mayores, pero si los costos de la financiación son mayores habrá destrucción de valor; a modo de ejemplo, se puede decir que si se implementa un proyecto con utilidad contable (sin considerar la financiación con recursos propios o ajenos) de 100 unidades monetarias (u.m.), pero para ello se invirtió 1000 u.m. a un costo de 25\%, no habrá creación de valor.

El sector pesquero peruano ha afrontado años de un mayor precio internacional de la harina de pescado el cual es un componente en el valor de las exportaciones y en las utilidades. Las empresas han realizado inversiones para obtener un producto de mayor calidad y enfrentan una situación en la cual la producción de harina de pescado depende del nivel de captura de la anchoveta.

Dentro de la estructura de costos se encuentran los derechos de pesca los cuales tiene relación positiva con el precio de la harina de pescado. Al respecto Galarza (2011) señala que en el sector pesquero el derecho de pesca es el $0,25 \%$ del precio FOB de la harina de pescado por tonelada de anchoveta, de un lado se concluye que en Perú el derecho de pesca es $0,25 \%$ del precio FOB de la harina y otras cargas regulatorias, siendo mayores a las que se paga en Chile, se aprecia que el precio de la harina es un componente del costo para las empresas pesqueras 
Apoyo Consultoría (2018).

El problema observado se refiere a que el precio es un componente del ingreso de las empresas, pero en este contexto también lo es de los costos por lo que surge la interrogante si dicha variable tiene una relación negativa con la creación de valor.

Por tal razón se requiere conocer la relación que existe entre el precio de la harina de pescado con el valor económico agregado EVA, tomando en cuenta otras variables importantes cómo la inversión realizada y el nivel de captura de anchoveta. En este estudio se evalúa a la empresa AGSAA quien ocupa la cuarta posición en la clasificación de empresas con mayor captura de recursos hidrobiológicos por embarcación y se dispone de información financiera al cotizar en la Bolsa de Valores de Lima. La empresa forma parte del grupo económico Austevoll Seafood AS, la cual lista en la Bolsa de Oslo.

Por lo anteriormente expuesto, el estudio tuvo como objetivo determinar el efecto del precio internacional de la harina sobre el valor económico agregado; considerando que es una forma de conocer la creación de valor en las empresas, siendo muy importante para la toma de decisiones en la empresa.

Las razones financieras construidas con información de estados financieros contables se vienen reemplazando por otras que midan la creación de valor para una mejor toma de decisiones. Las razones tradicionales no incorporan el costo del capital, ni el riesgo del sector, no es suficiente que una empresa obtenga utilidades para que la administración de la empresa cree valor para los accionistas.

\section{MATERIALES Y MÉTODOS}

Para la validación empírica de la hipótesis estudiada, se implementó un modelo teórico en el cual el EVA es explicado por tres variables independientes. La primera variable independiente es el precio internacional de la harina de pescado, se asume que los efectos de los incrementos de esta variable afecta positivamente en el EVA de Austral Group; se presume que, el incremento en el nivel de precios de la harina de pescado llevará a que las empresas pesqueras incrementen su niveles de pesca, lo que podría llevar a la depredación del recurso por lo que, para evitar esta situación, el Gobierno Peruano emitió el Decreto Legislativo 1084 (2008-06-26) conocida como la Ley sobre límites máximos de captura por embarcación, mediante la cual se establece un límite de captura de anchoveta en temporadas de pesca. De otro lado, para poder adaptarse a las exigencias de los mercados internacionales, las empresas pesqueras deben invertir en equipos; en este sentido, la segunda variable que explicaría el comportamiento del EVA es el nivel de capital invertido. La tercera variable es el nivel de captura, mientras mayor sea el nivel de captura se espera que mayor sea el nivel de producción de harina de pescado

La técnica econométrica utilizada para la estimación es el método de mínimos cuadrados ordinarios. La relación entre la creación de valor y el precio internacional de la harina se asume lineal.

En este estudio, los datos utilizados corresponden a series de tiempo trimestral desde el año 2009 a diciembre del 2018. El método econométrico utilizado es el de mínimos cuadrados ordinarios. El objetivo de esta investigación es conocer la relación que existe entre la creación de valor, la variable dependiente y el precio de la harina de pescado, variable independiente, el nivel de captura, variable independiente, y capital invertido, variable independiente. La relación funcional se asume lineal, por lo que los signos de los coeficientes de las variables independientes indicarían si la relación es directa (si el signo es positivo) o inversa (si el signo es negativo); si el coeficiente fuere estadísticamente no significativo, implicaría independencia de las variables.

Los valores calculados para las variables fueron estimados a partir de los siguientes datos:

$$
E V A=N O P A T-\text { WACC } \cdot \text { Caplnv }
$$

Donde:

NOPAT $=$ Utilidad operativa neta de impuestos

WACC $=$ Costo del capital invertido

Caplnv = Capital invertido

Austral Group SAA es una empresa con cotización pública, este hecho ha permitido obtener sus reportes financieros presentados en la Bolsa de Valores de Lima desde el año 2009 al 2018.

Las variables independientes en esta investigación han sido calculadas a partir de los siguientes datos: 
$\mathrm{X}_{1}=$ Precio de la harina de pescado en el período 2009-2018

Es la cotización que se obtiene de la Bolsa de Valores de Hamburgo en la cual se publican los precios de cierre de mes para los exportadores de harina de pescado se utiliza el precio promedio trimestral en soles.

$\mathrm{X}_{2}=$ Capital invertido en el período 2009-2018

Comprende el nivel de capital invertido trimestral es decir activos corrientes, activo fijo en maquinaria, equipo, inmuebles e intangibles en soles, según los estados financieros de la empresa, que no poseen costo de financiamiento.

$\mathrm{X}_{3}=$ Nivel de captura de anchoveta en el período 2009-2018

Es el nivel de captura de anchoveta en toneladas trimestrales valorizada en soles que captura la empresa para convertirla en harina de pescado según información del Ministerio de la Producción.

El modelo

El nivel observado del EVA viene determinado por los valores contables de las variables del lado derecho de la ecuación (1); sin embargo, en este estudio se ha postulado que existen variables subyacentes $\left(X_{1}, X_{2}, X_{3}\right)$ que son las que influyen en el comportamiento de la variable dependiente (EVA). En ese sentido, la relación a estimar se presenta en la siguiente ecuación:

$$
E V A_{t}=a+b \cdot X_{1, t}+c \cdot X_{2, t-1}+d \cdot X_{3, t}+u_{t}
$$

La ecuación (2) representa una relación lineal entre el EVA y las variables independientes. A diferencia de la ecuación (1), la cual es exacta por provenir de una relación contable, la (2) refleja una relación estocástica, por lo que al final se agrega la perturbación aleatoria

Este modelo midió la relación entre el precio internacional de la harina de pescado y la creación de valor en Austral Group, medido a través de su EVA. Se sugiere trabajar el capital invertido con un periodo de rezago como lo indica Chu (2015) por ser un mejor indicador, el cual impacta en la creación de valor. Lo señalado refleja el hecho de que todas las variables sean contemporáneas a excepción del capital invertido que se reflejará a valores del período previo; esto lleva a estimar el modelo conforme a la siguiente ecuación:

$$
E V A_{t}=a+b \cdot P I_{t}+c \cdot C a p l n v_{t-1}+d \cdot C A P_{t}+u_{t}
$$

\section{RESULTADOS}

El Modelo para analizar la relación entre el precio internacional de la harina de pescado y la creación del valor en Austral Group SAA es el siguiente:

$$
E V A_{t}=a+b \cdot P I_{t}+c \cdot C a p l n v_{t-1}+d \cdot C A P_{t}+u_{t}
$$

Donde EVA $A_{t}$ es la Creación de valor EVA trimestral

Para tal efecto se ha realizado el cálculo del EVA como lo indica Amat (2002) y lo aplica Pacheco (2009) es decir:

$E V A=$ NoPat - Costodecapital.Activo

NoPat es la Utilidad operativa neta de impuestos, es decir:

NoPat $=$ Utilidad operativa. $(1-\mathrm{t})$ donde $\mathrm{t}$ es la tasa impositiva.

Para Austral Group SAA se utilizaron sus estados financieros trimestrales y se realizó el cálculo respectivo tomando en cuenta la información desde el primer trimestre 2009 hasta el cuarto trimestre del 2018 lográndose tabular 40 observaciones.

Respecto al Costo del capital definido como WACC, es decir el costo promedio ponderando el costo de financiarse con acreedores y accionistas:

$W A C C=W e \cdot K e+W d \cdot K d \cdot(1-t)$ 
Donde:

We es peso del aporte del patrimonio, $\mathrm{Wd}$, es el peso de la deuda en la estructura de financiamiento, t es la tasa del impuesto a la renta y Kd se obtuvo de dividir el gasto financiero entre los pasivos con costo (sin incluir impuestos, proveedores y similares)

Ke es la rentabilidad exigida por los accionistas, la cual se obtuvo de la siguiente manera:

$$
K e=i l r+b . \text { Prima de riesgo }+ \text { Triesgo }
$$

ilr es tasa libre de riesgo considerando el rendimiento de los US\$ T Bond de 10 años en su media geométrica que se considera consistente con la metodología estadística acorde con las características del mercado local e internacional tal como señalan Fuertes y Inouye (2006)

Tomando en cuenta estadísticas Damodaran (2018) se considera ilr $=3.86 \%$

De otro lado, Fuertes e Inouye (2006), afirman que para la prima de riesgo de mercado se sugiere trabajar con un valor promedio de $4 \%$ o menos debido a los recientes estudios sobre este indicador y tomando en cuenta la dinámica de los mercados reales, para lo cual la data de Damodaran (2018) nos muestra un valor de 4,24\%. Triesgo es el riesgo país, cuya información se ha obtenido de las estadísticas semanales del Banco Central de Reserva. Con la información de los componentes del WACC se pueden mostrar los resultados anuales y trimestrales para la empresa en estudio $\mathrm{Pl}_{t}$ es el precio internacional promedio trimestral de la harina de pescado obtenido de las notas semanales del Banco Central de Reserva indicando como fuente a Reuters y Bloomberg (2018), Es la cotización internacional de la harina de pescado en los mercados internacionales.

$I N V_{t}$ es el nivel de capital invertido trimestral que realiza la empresa, el cual es el capital invertido. Dicho valor se obtiene del total de activos deduciendo aquella parte cuya fuente de financiamiento no tiene costo de financiamiento. Se entiende por financiamiento a los recursos obtenidos de accionistas y acreedores que requieren el costo financiero. $C A P_{t}$ es el nivel de captura de anchoveta trimestral que realiza la empresa expresado en toneladas, este valor se obtuvo tomando en cuenta los niveles de captura de anchoveta trimestral para cada año del sector, el cual se multiplicó por el nivel de participación de cada empresa en estudio.

En el modelo no se aplicaron logaritmos ni diferencias, pero se utilizaron rezagos en determinadas variables, se logró superar la autocorrelación, la heteroscedasticidad y la multicolinealidad entre las variables y se incorporan variables dummy para tomar en cuenta el Fenómeno del Niño y alzas en el precio de la harina en determinados meses.

\section{Estimación del modelo}

$E V A_{t}=a+b \cdot P I_{t}+c \cdot C a p l n v_{t-1}+d \cdot C A P_{t}+u_{t}$

\section{Análisis de resultados para EVA en Austral Group}

Dependent Variable: EVA

Method: LeastSquares

Date: $07 / 21 / 19$ Time: $21: 16$

Sample (adjusted): 2009Q2 2018Q4

Includedobservations: 39 afteradjustments

La variable precio es estadísticamente significativa al 90\% de confianza e incide negativamente en el EVA. Las variables capital invertido y captura -rezagada un periodo- impactan positivamente en el EVA y son estadísticamente significativas al $95 \%$ y $99 \%$ de confianza respectivamente. Además, el EVA resulta autorregresivo en un periodo al $95 \%$ de confianza. La variables dummy 3 ( D3) y 4 ( D4) referidas a variaciones de la captura en periodos específicos son significativas al $99 \%$ de confianza y muestran el signo esperado.

Las variables independientes explican la variabilidad del EVA en más del $60 \%$ de acuerdo al $R 2$ ajustado.

El modelo estimado es válido en su conjunto (P-valor de prueba $\mathrm{F}$ menor a 0.05). 
Tabla 1

Efectos del Precio de la harina de pescado, capital invertido y captura sobre EVA en AGSAA 2009-2018

\begin{tabular}{|c|c|c|c|c|}
\hline Variable & Coefficient & Std. Error & t-Statistic & Prob. \\
\hline C & -63883103 & 48488287 & $-1,317496$ & 0,1973 \\
\hline PRECIO & $-12832,40$ & 6850,006 & $-1,873341$ & 0,0705 \\
\hline PRECIO(-1) & 3890,495 & 6488,563 & 0,599593 & 0,5531 \\
\hline CAPITAL & 0,110365 & 0,048355 & 2,282386 & 0,0295 \\
\hline CAPTURA(-1) & 0,022054 & 0,008012 & 2,752704 & 0,0098 \\
\hline EVA $(-1)$ & $-0,276509$ & 0,121419 & $-2,277317$ & 0,0298 \\
\hline D3 & -50308576 & 16859382 & $-2,984011$ & 0,0055 \\
\hline D4 & 38399289 & 10343872 & 3,712274 & 0,0008 \\
\hline R-squared & 0,683536 & Mean dependentvar & & 3214452 \\
\hline Adjusted R-squared & 0,612077 & S.D. dependentvar & & 26001903 \\
\hline S.E. of regression & 16194894 & Akaikeinfocriterion & & 36,21897 \\
\hline Sum squaredresid & $8.13 E+15$ & Schwarzcriterion & & 36,56022 \\
\hline Log likelihood & $-698,2700$ & Hannan-Quinncriter. & & 36,34141 \\
\hline F-statistic & 9,565356 & Durbin-Watson stat & & 1,894428 \\
\hline Prob(F-statistic) & 0,000003 & & & \\
\hline
\end{tabular}

Las siguientes variables dummy no resultaron estadísticamente significativas.

D1: Periodo de incremento de precio internacional 2014.3 al 2016.4

D2: Fenómeno Niño Costero, se incrementan las capturas: 2016.3 al 2017.2

\section{DISCUSIÓN}

Chu (2015) afirma que el sistema de información contable no incorpora el concepto de costo de oportunidad de los inversionistas. El autor observa que las metodologías que más se han empleado y aplicado en investigaciones sobre la medición de la creación de valor es el , el valor de mercado es valor presente por definición.

Desde una perspectiva financiera Rodríguez (2017) considera al EVA como un indicador financiero, el cual simboliza el valor de mercado. Es una guía para proporcionar recursos a las inversiones que sean capaces de producir un rendimiento mayor al costo de capital, las inversiones son los activos netos que son productivos en periodos posteriores a su adquisición incluyendo el capital de trabajo y el costo del capital que se aplica a dichos activos.

El modelo alternativo para explicar los determinantes de la creación de valor es seguir a Álvarez (2016) quien señala que para calcular el EVA, se requiere conocer la utilidad operacional luego de impuestos la cual se expresa en una determinada moneda para lo cual se requiere conocer el capital invertido por su respectivo costo en la misma unidad monetaria.

La literatura revisada nos mostraba la importancia de medir la creación de valor usando EVA y se formulaba al precio internacional de la harina, el capital invertido y el nivel de captura como las variables explicativas del modelo. Los resultados de la investigación nos permiten hallar la relación negativa entre el precio internacional de la harina de pescado y la creación de valor EVA, El precio de la harina es un componente de las ventas pero también los derechos de pesca de la empresa en estudio.

Desde el año 2013 la empresa realizó mayores inversiones en activo fijo para mejorar la calidad del producto, se encuentra que el capital invertido tiene relación positiva con la creación de valor EVA nivel estadístico significativo.

Respecto a la relación entre creación de valor EVA y el nivel de captura la relación es positiva, estadísticamente significativa pero su impacto es pequeño. Se podría aumentar el nivel de captura si se aprueba una norma para que 
las embarcaciones de las empresas del sector puedan pescar otras especies hidrobiológicas.

Desde el cuarto trimestre del 2017 se aprecia una recuperación del precio de la harina y con un mayor nivel de captura, sin embargo, los costos que enfrenta la empresa son mayores lo cual se refleja en una menor utilidad operativa, por ende, en un menor NoPat. La empresa realiza un mayor esfuerzo en reducir el costo del capital invertido y el mismo es mayor buscando la diversificación en productos frescos y congelados para incrementar los ingresos.

Aunque no ha sido investigada la relación entre precio de la harina y creación de valor, si lo ha sido la relación entre dicho precio y los costos de las empresas pesqueras. En ese sentido, los trabajos de Galarza (2011) y Apoyo (2018), aportan evidencia empírica a esta relación, por cuanto se aprecia una relación positiva dado que si el precio de la harina se incrementa también ocurre con los costos y por ende reduce los beneficios de las empresas.

Se recomienda continuar con la diversificación en los productos exportables y darle valor agregado a la harina de pescado, por ejemplo, mediante la producción de alimentos balanceados para la industria de la Acuicultura que experimenta un importante crecimiento en el mundo.

Se requiere incrementar la eficiencia de la empresa y reformular los derechos de pesca en el sector, para lo cual se debería aplicar un porcentaje a la tonelada capturada, condicionada a la generación de utilidad operativa. El precio de la harina y exportables de valor agregado deberían enfocarse como componentes en la creación de valor en las empresas del sector pesquero.

\section{REFERENCIAS BIBLIOGRÁFICAS}

Álvarez, I. (2016). Finanzas estratégicas y creación de valor, Bogotá: Ecoe Ediciones. pp. 91-92.

Amat, O. (2019). Valoración y compra venta de empresas. Barcelona: Editorial Profit. pp. 75-76.

Apoyo Consultoría. (2018). Análisis del desempeño financiero, de los costos regulatorios y tributarios y de la fijación de derechos de pesca en el Perú, Informe presentado para Sociedad Nacional de Pesquería. Lima: Sociedad Nacional de Pesquería. Recuperado de :https://www.snp.org.pe/wp-content/uploads/2019/10/2018-09-18-Apoyo-Informe-desempe\%C3\%B1o-financiero.pdf

Chu, M. (2015). La creación de valor en las finanzas, mitos y paradigmas. Lima: Universidad Peruana de Ciencias Aplicadas. pp. 31-32.

Damodaran, A (2018). Damodaran on line. Nueva York, EEUU: Recuperado de http://pages.stern.nyu.edu/ adamodarl

Decreto Legislativo № 1084, Ley sobre límites máximos de captura por embarcación. Congreso de la República: Lima. 26 de junio del 2008.

Fuertes, A., \& Inouye, G. (2006). Tasa libre de riesgo y prima por riesgo de mercado en el modelo Capm.. Lima: Sociedad Administradora de Fondos de Inversión Popular. pp. 18-22.

Galarza, E., \& Collado, N. (2013). Los derechos de pesca: el caso de la industria pesquera de anchoveta. Apuntes. Revista de Ciencias Sociales, 40(73), 7-42. Recuperado de http://revistas.up.edu.pe/index.php/apuntes/article/view/686

Gomez-Besare, F., Madariaga, J., \& Santibañez, J, (2013). Modelos integrados de análisis financiero. Barcelona: Harvard-Deusto Finanzas \& Contabilidad, pp. 141-14.

Gujarati, D., \& Porter, D. (2010). Econometría. 5ta edición. México D.F.: Mc Graw Hill. pp. 37-39.

FAO. (2020). El estado mundial de la pesca y la acuicultura 2020. La sostenibilidad en acción. Roma: FAO. Recuperado de https://doi.org/10.4060/ca9229es.

Pacheco, R. (2010). El EVA y la creación de valor en las empresas peruanas, periodo 1999-2003. (Tesis para optar el grado de doctor en Economía). Universidad Nacional Mayor de San Marcos, Lima. pp. 8-9.

Rodríguez, F. (2017). Finanzas 2 Finanzas Corporativas: una propuesta metodológica: México D.F.: Instituto Mexicano de Contadores Públicos. pp. 47-48.

Correo electrónico: jcor67@gmail.com 